\title{
Identification of Significant Pathways Induced by PAX5 Haploinsufficiency Based on Protein-Protein Interaction Networks and Cluster Analysis in Raji Cell Line
}

\author{
Jia Gu, TongJuan Li, Lei Zhao, Xue Liang, Xing Fu, Jue Wang, Zhen Shang, \\ Wei Huang, and Jianfeng Zhou \\ Department of Hematology, Tongji Hospital, Tongji Medical College, Huazhong University of Science and Technology, \\ Wuhan, Hubei, China
}

Correspondence should be addressed to Wei Huang; 2896607051@qq.com

Received 6 November 2016; Revised 23 January 2017; Accepted 30 January 2017; Published 21 February 2017

Academic Editor: Yudong Cai

Copyright (C) 2017 Jia Gu et al. This is an open access article distributed under the Creative Commons Attribution License, which permits unrestricted use, distribution, and reproduction in any medium, provided the original work is properly cited.

PAX5 encodes a transcription factor essential for B-cell differentiation, and PAX5 haploinsufficiency is involved in tumorigenesis. There were few studies on how PAX5 haploinsufficiency regulated genes expression to promote tumorigenesis. In this study, we constructed the cell model of PAX5 haploinsufficiency using gene editing technology in Raji cells, detected differentially expressed genes in PAX5 haploinsufficiency Raji cells, and used protein-protein interaction networks and cluster analysis to comprehensively investigate the cellular pathways involved in PAX5 haploinsufficiency. The clusters of gene transcription, inflammatory and immune response, and cancer pathways were identified as three important pathways associated with PAX5 haploinsufficiency in Raji cells. These changes hinted that the mechanism of PAX5 haploinsufficiency promoting tumorigenesis may be related to genomic instability, immune tolerance, and tumor pathways.

\section{Introduction}

The paired box domain gene 5 (PAX5) encodes a paired box domain (PBD) transcription factor essential for B-cell differentiation that activates crucial genes for B-cell lineage differentiation and represses genes important for commitment in other hematopoietic lineages $[1,2]$. Mutation of PAX5 participates in B-cell tumorigenesis [3, 4]. Conditional PAX5 deletion in mice allowed mature B-cells from peripheral lymphoid organs to dedifferentiate in vivo back to early uncommitted progenitors in the bone marrow. Mice lacking PAX5 in mature B-cells also developed aggressive lymphomas [5]. PAX5 haploinsufficiency synergized with STAT5 activation to initiate acute lymphoblastic leukemia (ALL) and the probability of tumor formation was $100 \%$ [6]. PAX5 haploinsufficiency cooperated with BCR-ABL1 to induce acute lymphoblastic leukemia [1].

PAX5 has been reported as being frequently altered in both childhood [2] and adult [7, 8] B-ALL. PAX5 mutation was also reported in both Hodgkin lymphoma [9] and non-Hodgkin lymphoma [10, 11]. PAX5 genomic deletions were predicted to result in PAX5 haploinsufficiency or expression of PAX5 isoforms with impaired DNA binding [7, 12, 13], which resulted in PAX5 haploinsufficiency. So, PAX5 haploinsufficiency plays an important role in lymphocytic neoplasm.

We do not know how PAX5 haploinsufficiency regulates genes expression to promote tumorigenesis, though previous studies showed heterozygous mice $(\mathrm{PAX} 5+/-)$ had higher penetrance of B-ALL than wild-type mice (PAX $5+/+)$ $[14,15]$. The transcription factor PAX5 is pivotal for Bcell commitment in mice. It represses lineage-inappropriate gene expression while concurrently activating the B-cell gene expression program. Clare Pridans performed global gene expression screen of wild-type (PAX5+/+) and PAX5deletion (PAX5-/-) pro-B-cells in an attempt to identify the crucial PAX5 targets in early B-lymphopoiesis. He identified 109 PAX5 targets comprising 61\% activated and 39\% 
repressed genes [16]. A key function of PAX5 is to activate secondary transcription factors that further reinforce the B-cell program [16]. The allele-specific regulation of PAX5 is random, reversible, and independent of parental origin and correlates with synchronous replication during B-cell development. The allele-specific regulation of PAX5 may be a common mechanism causing the haploinsufficiency and frequent association of other PAX genes with human disease [17]. Cell model of PAX5 haploinsufficiency is more similar to the human disease state, and PAX5 haploinsufficiency (PAX5+/-) may have different effect on target genes compared with PAX5-/- at cell level, but there were few studies on PAX5 haploinsufficiency on genes expression at the cellular level.

In our study, we constructed a new cell model of PAX5+/using gene editing technology which knocked out one PAX5 allele in Raji cell line (lymphoblastoid cell line derived from Burkitt lymphoma). We analyzed the gene expression profile in PAX5+/- Raji cells and their mother wild-type cells. We constructed the protein-protein interaction (PPI) network of the differentially expressed genes and screened out the most significant subnetwork. In addition, the enriched functions and pathways of DEGs were used to identify significant pathways involved in PAX5 haploinsufficiency Raji cells. This study was very valuable for our understanding of how PAX5 haploinsufficiency regulated genes expression to promote tumorigenesis.

\section{Materials and Methods}

2.1. Cell Culture. The lymphoblastoid cell line Raji was purchased from China Center for Type Culture Collection (CCTCC, Wuhan, China) and HEK293T was purchased from the American Type Culture Collection (ATCC, United States). Raji cells were cultured in RPMI1640 medium containing 5 10\% fetal calf serum (FCS, Invitrogen, United States), and HEK293T cells were cultured in DMEM supplemented with $10 \%$ dialyzed fetal bovine serum.

\subsection{Construction and Validation of Customized CRISPR/CAS9} Expression Vectors. The vector pSpCas9(BB)-2A-GFP (PX458) (Plasmid \#48138) was purchased from Addgene (Massachusetts, USA). The oligo-DNA targeting the PAX5 exon5 locus was designed on the MIT online software ZhangFeng lab: http://crispr.mit.edu/. We selected three high scored sequences and designed their respective complement chains with restriction site; each single strand oligo-DNA chain was synthesized in Invitrogen company as follows:

PAX5 gRNA-F1: cacc GACAAAAGTACAGCAGCCAC

PAX5 gRNA-R1: aac GTGGCTGCTGTACTTTTGTC

PAX5 gRNA-F2: cacc AACCAACCAGTCCCAGCTTC

PAX5 gRNA-R2: aaac GAAGCTGGGACTGGTTGGTT
PAX5 gRNA-F3: cacc ACCAACCAGTCCCAGCTTCC

PAX5 gRNA-R3: aac GGAAGCTGGGACTGGTTGGT

Next, we annealed the two complement chains to form dsDNA using Precut sgRNA Cloning kit and pSD-gRNA Plasmid construction Kit (Biomics Biotechnologies Co., Ltd., Jiangsu, China) according to the instruction. This was followed by BbsI digestion and ligation with T4 ligase to construct Cas9/sgRNA plasmids targeting PAX5. The Cas9/sgRNA plasmids were amplified, purified with EndoFree Plasmid Maxi Kit (QIAGEN, Germany), and validated by sequencing. The Cas9/sgRNA plasmids were electrotransfected into HEK293T cells. The T7 Endonuclease I assay was applied to measure the NHEJ-mediated mutations efficiency in the endogenous PAX5 gene. The most efficient Cas9/sgRNA plasmid (gRNA-F1/R1) was chosen for subsequent research.

2.3. Construction and Identification of PAX5+/- Raji Cell Clone. $5 \times 10^{5} \sim 2 \times 10^{6}$ Raji cells in good condition were collected and suspended with matched solution supplemented with $5 \mu \mathrm{g}$ CRISPR/CAS9 plasmid. Electrotransfection was performed with optimized program on LONZA 4D Nucleofector System (Lonza, Switzerland). Cells were cultured for $48 \mathrm{~h}$ and then sorted with Beckman MoFlo XDP (Beckman Coulter, Inc., USA), aiming to select cells with high GFP expression. The sorted cells were seeded in 96-well plates in the manner of single cell. Two or three weeks later, cells were collected for identification. Genomic DNA was isolated using the DNA Isolation Kit (BioTeke Corporation, Beijing, China) according to the manufacturer's instructions. PCR was used to amplify the PAX5 gene for mutation analysis. The PCR primers, synthesized by Sangon Biotech (Shanghai, China), were as follows: forward primer CTTCAGAAGAGGCACTTGAAGC and reverse primer TTACCAGGTTCAGCCCTTGG. The PCR product was reclaimed for sequence determination. The sequencing results were compared with the published PAX5 gene sequence to determine the presence of pax5+/variants.

2.4. Western Blot Analysis for PAX5. Cells were lysed with RIPA lysis buffer (Beyotime, China) supplemented with a protease inhibitor cocktail (Roche, Switzerland). The bicinchoninic acid protein assay (Thermo Scientific, USA) was used to measure protein concentration. $40 \mu \mathrm{g}$ of total lysate was subjected to SDS-PAGE and then transferred to nitrocellulose membranes (Bio-Rad Laboratories, USA). The membranes were incubated with the antibodies against PAX5 and GAPDH purchased from Abcam Biotechnology (Abcam, CA, USA), and then they were blotted with corresponding HRP-linked secondary antibodies. The proteins were detected using an enhanced ECL system (Pierce, USA). Quantification was performed with ImageJ (https://imagej.nih.gov/) and each sample's ratio relative to the loading control GAPDH was calculated. 
2.5. Quantitative RT-PCR Analysis. Total RNA was extracted with TRIzol (Invitrogen, USA) from the wide-type and mutational cells. A NanoDrop microvolume spectrophotometer (Thermo Fisher) was used to quantify the RNA and RTPCR was performed with $2 \mu \mathrm{g}$ of total RNA and oligo-dT, and $1 \mu \mathrm{L}$ of cDNA was used as quantitative RT-PCR template in $10 \mu \mathrm{L}$ PCR mix (GeneCopoeia, Guangzhou, China) with $1 \mu \mathrm{L}$ of primer and $8 \mu \mathrm{L}$ ddH2O $(20 \mu \mathrm{L}$ reaction volume). Quantitative RT-PCR was performed using a CFX96 Touch $^{\mathrm{TM}}$ Real-Time PCR Detection System (Bio-Rad) according to the instructed thermocycler program for each locus. PCR primer sequences, synthesized by BGI, Shenzhen, China, are shown as follows: GAPDH: $5^{\prime}$-GAGTCCACTGGCGTCTTCA-3' (forward), 5'-GGGTGCTAAGCAGTTGGT-3' (reverse); CD19: $5^{\prime}$-GGCCCGAGGAACCTCTAGT-3' (forward), 5' TAAGAAGGGTTTAAGCGGGGA-3' (reverse); CD79A: $5^{\prime}$-CAAGAACCGAATCATCACAGCC-3' (forward), $5^{\prime}$ TCTGCCATCGTTTCCTGAACA-3' (reverse); FCER2: $5^{\prime}$ CCAGGAATTGAACGAGAGGAAC-3 ${ }^{\prime}$ (forward), $5^{\prime}$-TTGATCCACTTTTCA GGGCAC-3' (reverse); IGLL1: $5^{\prime}$-ACCCAGCTCACCGTTTTAAGT-3' (forward), 5' -GGTCACCGT CAAGATTCCCG-3' (reverse).

2.6. Microarray Assay. Total RNA was exacted using TRIzol reagent (Invitrogen, USA) according to the manufacturer's protocol. The experimental samples' RNA integrity number (RIN) was confirmed to be no less than 7.0 to ensure the quality and quantity. Library construction and RNA sequencing were performed at Beijing Genomics Institute (BGI, Shenzhen). The final libraries were quantitated by the Agilent 2100 bioanalyzer instrument (Agilent DNA 1000 Reagents) to ensure the size and purity of the sample and then sequenced using the HiSeq 2000 System (TruSeq SBS KIT-HS V3, Illumina), with read length 50 .

After being aligned to the human reference genome (NCBI Build 36.1) using SOAPaligner-v2.21 software (BGI) with high-quality reads, the matched data were aligned with reference sequence on Human RefSeq mRNA (NCBI). We normalized the expression levels for each gene to the reads per kilobase of the target exon per million mapped reads (RPKM) to facilitate the comparison of transcripts between samples. The differentially expressed genes (DEGs) between each pair were identified using the standard of "FDR $\leq 0.001$ and the absolute value of $\log 2$ Ratio $\geq 2$."

2.7. The Motif Analysis of PAX5 and Identification of PAX5 Target Genes. The motif analysis of PAX5 and identification of PAX5 target genes were accomplished based on TRRUST Database (http://www.grnpedia.org/trrust/result .php?gene $=$ PAX5) and TRED Database (https://cb.utdallas .edu/cgi-bin/TRED/tred.cgi?process=searchTFGeneForm).

2.8. Construction of PPI Network and Subnetwork. The online database STRING (Search Tool for the Retrieval of Interacting Genes) offers uniquely wide coverage and ease of access to both laboratorial and predicted interaction information [18]. In our study, the interactions between DEGs were derived based on STRING and the associations with a correlation coefficient $>0.8$ were recognized as PPIs. The PPI network was constructed and visualized using Cytoscape software, as formerly described [19]. Cytoscape is an open software project for integrating biomolecular interaction networks with high-throughput expression data and other molecular states into a unified conceptual framework.

2.9. Pathway Enrichment Analysis. The DAVID (Database for Annotation, Visualization and Integrated Discovery) contains an integrated biological knowledge base and analytic tools, with the purpose of extracting biological meaning from large gene/protein lists [20]. The KEGG (Kyoto Encyclopedia of Genes and Genomes) is a knowledge base for the analysis of gene functions, connecting genomic information with higher order functional information [21]. In our study, pathway enrichment analysis was administered for the PPI network by DAVID and the significantly enriched pathways were confirmed with a value of $p<0.05$.

\section{Results}

3.1. Construction and Identification of PAX5+/- Raji Cells. Targeted genome editing tools such as CRISPR-Cas9 system have been widely used to modify genes in model systems including animal and human cells [22]. We designed a gRNA that directed exon 5 of PAX5, which was shared by all different transcripts, and we inverted it into the cas9 and GFP expressing vector. After the editing and subsequent screening process, we randomly picked out the clones and screened out mutated clones by DNA sequencing. Sequencing results showed that mutation clones had haploid deletion mutation in exon5 of PAX5 by comparison of DNA sequences of mutation clone with that of wild-type clone (Figure 1(a)). Haploid deletion mutation resulted in haploid termination of PAX5 mRNA transcription. Western blot showed that PAX5 protein level in mutation clone was significantly lower than that in wild-type clone $(p<0.05)$ (Figure $1(b))$.

3.2. PAX5 Haploinsufficiency Induced the Differentially Expressed Genes (DEGs). After analyzing the microarray data in PAX5+/- Raji cells and their mother wild-type cells, we screened out a total of 213 DEGs in PAX5+/- Raji cells (MUT1) compared to mother wild-type cells, including 82 downregulated genes and 131 upregulated genes. A total of 199 DEGs were screened out in PAX5+/- Raji cells (MUT2) compared to mother wild-type cells, including 85 downregulated genes and 114 upregulated genes. There were 135 common target genes, including 47 downregulated genes and 88 upregulated genes.

3.3. The Motif Analysis of PAX5 and Identification of PAX5 Target Genes. Based on motif analysis of PAX5, we searched out 28 PAX5 target genes, that is, BAX, BCL2, BLK, CCND1, CD19, D79A, CDKN1A, FCER2, FHL2, LEF1, MET, MMP1, MYCNA, PRDM1, PRKCE, RAG2, RB1, TIMP1, TP53, XBP1, EGR1, ESR1, ELK1, ETS1, KCNH4, VPREB1, IGLL1, and KCNH8. Two differentially expressed genes (IGLL1 and 

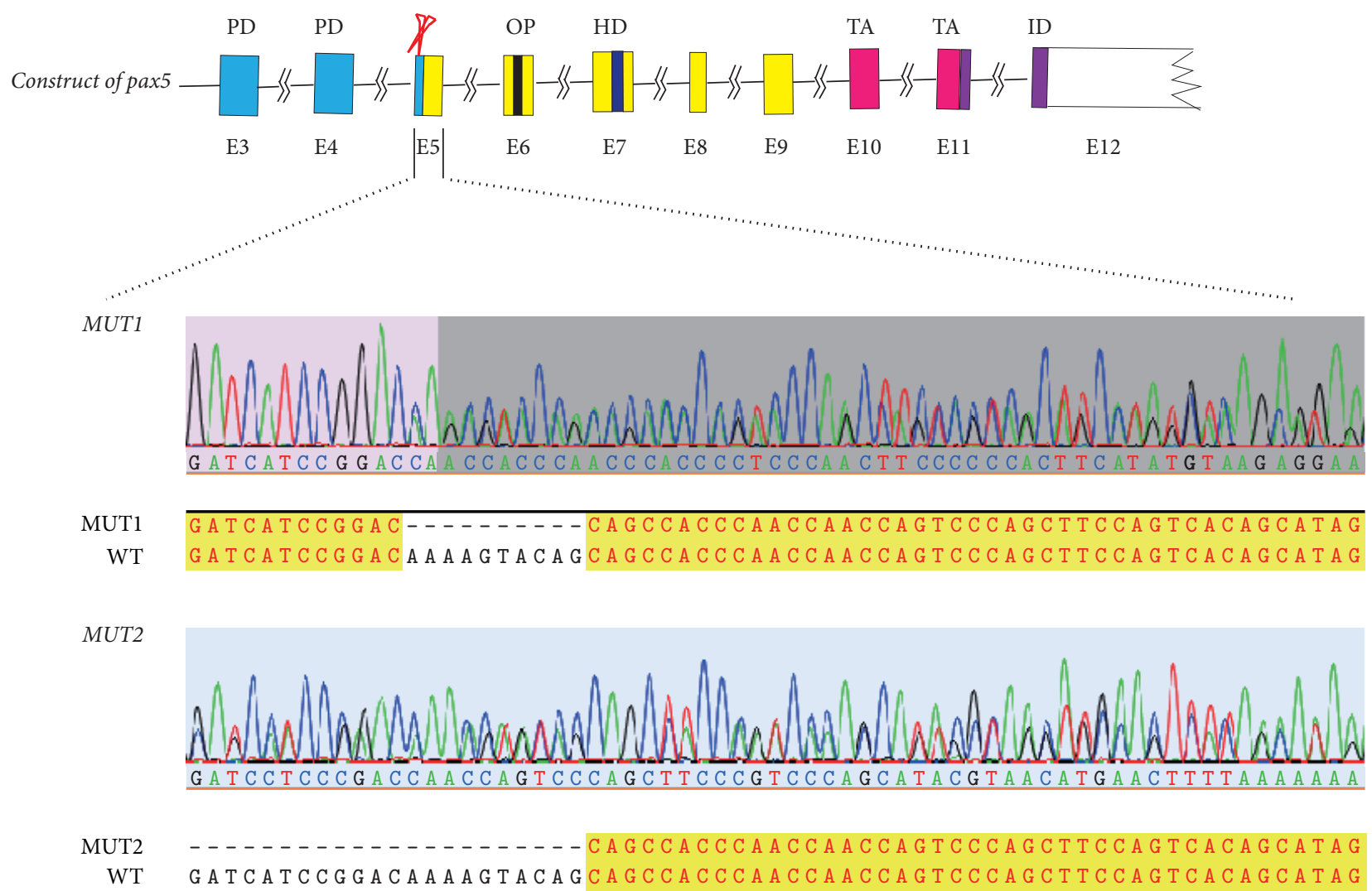

(a)
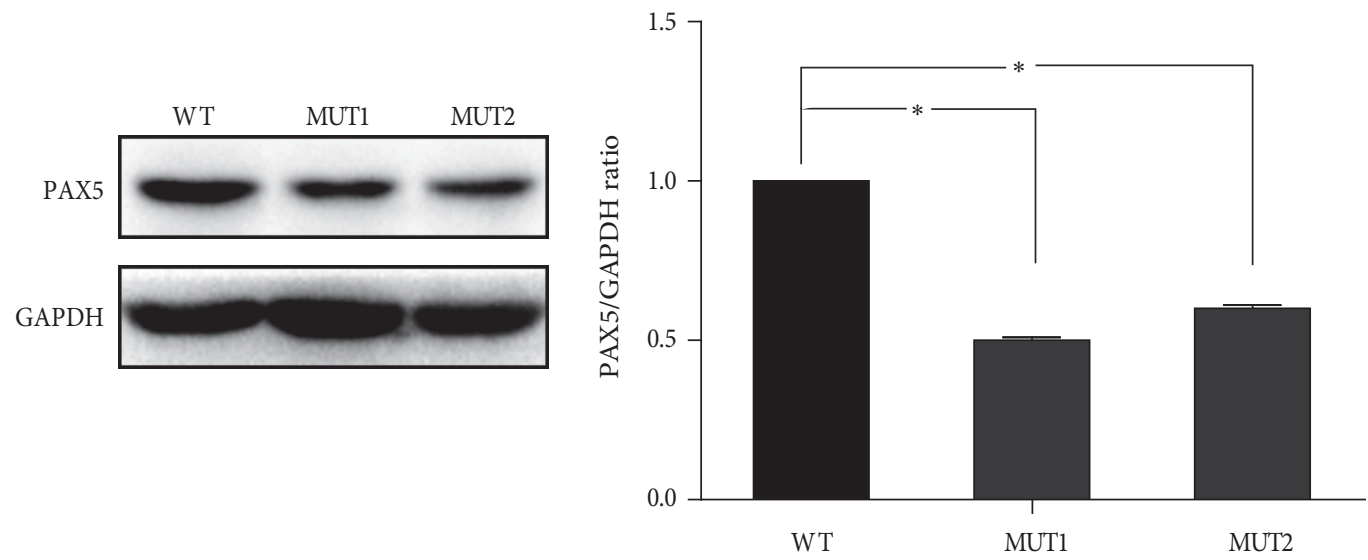

(b)

FIgURE 1: Mutation type of pax 5 and identification. (a) The construction of pax 5 and mutation type of pax 5 . E5 was the target of gene editing operation, and sequencing analyses identified knocked out regions of E5. (b) Protein level of pax 5 tested by western blot. Protein level of pax 5 in MUT1 and MUT2 was less than 50\% of that in WT. E: exon of pax5; PD: paired box domain; OP: octapeptide domain; HD: homeodomain; TA: transactivation domain; ID: inhibitory domain; WT: wild-type clone; MUT: mutation clone. $*$ means $p<0.05$.

FCER2) were identified as targets of PAX5. The expression of IGLL1 and FCER2 was decreased in PAX5+/- Raji cells. CD19 and CD79A are recognized target genes of PAX5, but we found that the expression of CD19 and CD79A was not changed from the microarray data. In order to verify the results, we detected mRNA level of CD19, CD79A, IGLL1, and FCER2 using quantitative PCR. Results showed that the expression of IGLL1 and FCER2 was decreased and the expression of CD19 and CD79A was not changed (Figure 2).

3.4. Construction of PPI Network. There were 135 common target genes, which were critical to explore the potential roles of PAX5 haploinsufficiency. The analysis of PPI networks found that there were 49 genes which were related to each 


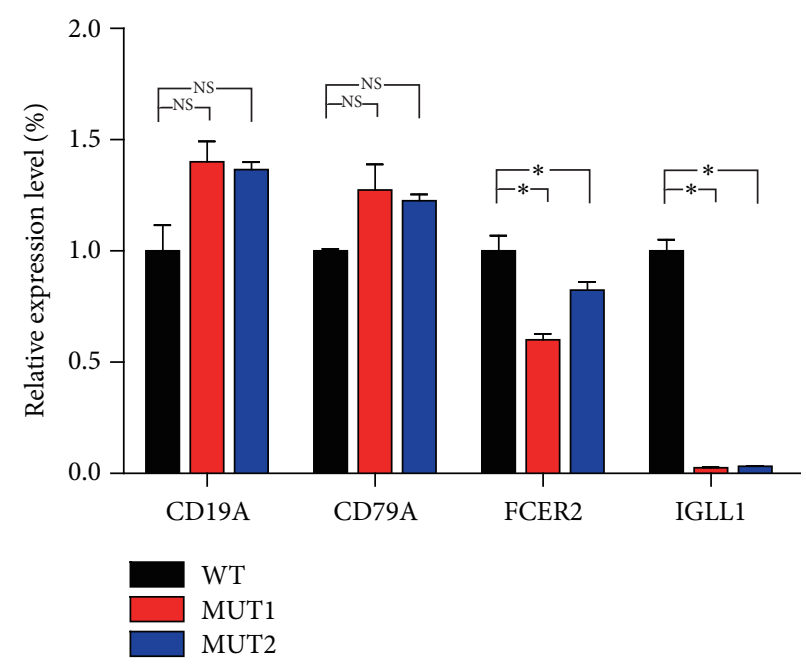

FIGURE 2: Target genes tested by quantitative RT-PCR. The expression of CD19 and CD79A was not changed. The expression of IGLL1 and FCER2 was decreased. ${ }^{\mathrm{NS}} p>0.05$ versus $\mathrm{WT},{ }^{*} p<0.01$ versus WT.

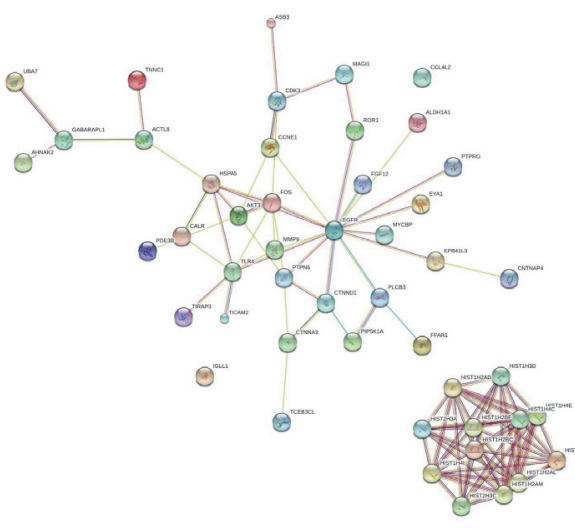

(a)

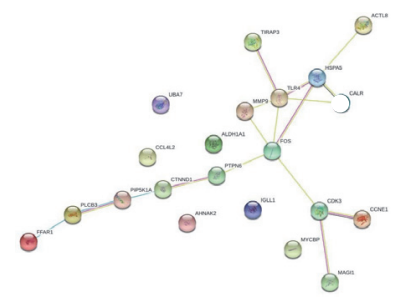

(b)

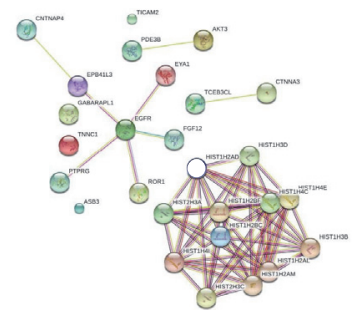

(c)

FIGURE 3: The differentially expressed genes were used to search the STRING database to predict their protein-protein interactions in pax5 mutation cells. In the network shown, the nodes are the proteins and the lines represent the predicted functional associations. The number of lines indicates the strength of the predicted functional interactions of the proteins. (a) Network of differentially expressed genes (DEGs). (b) The downregulated DEG PPI subnetwork. (c) The upregulated DEG PPI subnetwork.

other. The total DEGs PPI network contained 39 nodes and 157 edges (interactions), including 21 downregulated DEGs and 28 upregulated DEGs (Figure 3(a)). The downregulated DEG PPI subnetwork contained 21 nodes and 17 edges (Figure 3(b)). The upregulated DEG PPI subnetwork contained 28 nodes and 61 edges (Figure 3(c)). These three networks indicated that PAX5 haploinsufficiency greatly disturbed the PPI network in Raji as DEGs interactions change the biological consequences. We found all histones constructed one unattached network, and there were several important node genes such as EGFR, FOS, HSPA5, TLR4, and MMP9 (Figure 3).
3.5. Functional Annotation Clustering of DEGs in Network Using the DAVID. Using the DAVID, we did functional annotation cluster analysis for 49 interconnected genes. DAVID functional clustering of 49 genes returned 18 clusters, and there were 5 clusters with enrichment scores $>1$ $(p<0.05)$ (Table 1). These significant clusters reflected the notion that the dataset was representative of basic biological processes. PAX5 haploinsufficiency had the greatest influence on clusters involved in gene transcription, inflammatory and immune response, and cancer pathways. There were 17 genes involved in gene transcription, including 12 histone cluster genes. Six genes of DEGs in network participated 
TABLE 1: Functional annotation clustering in the DAVID disease/cancer database.

\begin{tabular}{|c|c|c|c|}
\hline Cluster & Count & Score & Genes \\
\hline Gene transcription & 17 & 2.31 & $\begin{array}{l}\text { HIST2H3A, HIST1H2BC, HIST1H2BF, HIST1H2AD, } \\
\text { HIST1H3B, HIST1H4E, HIST1H3D, HIST1H4C, } \\
\text { HIST1H2AM, HIST1H4I, HIST1H2AL, HIST2H3C, EGFR, } \\
\text { FOS, CALR, EYA1, MAGI1 }\end{array}$ \\
\hline $\begin{array}{l}\text { Inflammatory and } \\
\text { immune response }\end{array}$ & 6 & 1.42 & TICAM2, CCL4L2, TLR4, FOS, AKT3, PTPN6 \\
\hline Pathways in cancer & 7 & 1.39 & EGFR, CCNE1, FOS, MMP9, FGF12, AKT3, CTNNA3 \\
\hline Cell adhesion & 7 & 1.38 & $\begin{array}{l}\text { EGFR, CNTNAP4, MAGI1, CTNND1, CCL4L2, CTNNA3, } \\
\text { PTPN6 }\end{array}$ \\
\hline Protein phosphatase & 7 & 1.34 & EGFR, PTPN6, EYA1, PTPRG, ROR1, AKT3, CDK3 \\
\hline Tight junction & 4 & 1.03 & EPB41L3, MAGI1, AKT3, CTNNA3 \\
\hline
\end{tabular}

in inflammatory and immune response. Seven genes of DEGs in network participated in pathways in cancer. Other clusters included cell adhesion, protein phosphatase, and tight junction.

\section{Discussion}

PAX5 haploinsufficiency occurred frequently in lymphocytic neoplasm and plays an important role in lymphocytic tumorigenesis. Our report described the characteristics of changed gene expression after PAX5 was haploidentically knocked out in Raji cells.

We obtained two PAX5+/- Raji cell clones by targeted genome editing tools. Western blot showed that PAX5 protein level in PAX5+/- Raji clones was significantly lower than that in wild-type Raji clone. Based on motif analysis of PAX5, two differentially expressed genes (IGLL1 and FCER2) were identified as targets of PAX5. The expression of IGLL1 and FCER2 was decreased in PAX5+/- Raji cells. CD19 and CD79A, which are recognized target genes of PAX5, were not changed from the microarray data. Quantitative PCR confirmed microarray data. Expression of PAX5 in pro-B cells activates the genes such as CD19 and CD79A which were required for differentiation toward B-cells. Ex vivo, PAX5-/pro-B cells are not restricted to the B-lymphoid lineage [23]. Clare Pridans performed a global gene expression screen of wild-type $(\mathrm{PAX} 5+/+)$ and PAX5-deletion (PAX5-/-) pro-B cells in an attempt to identify the crucial PAX5 targets. The expression of CD19 and CD79A was decreased in PAX5-/pro-B cells compared to that in PAX $5+/+$ pro-B cells [16], but there was no change in expression of IGLL1 and FCER2. Our report showed opposite results. The expression of CD19 and CD79A was not significantly decreased in PAX5+/- Raji cells compared to that in PAX5+/+ Raji cells, and the expression of IGLL1 and FCER2 was significantly decreased in PAX5+/Raji cells. So, we believe that the genetic variation characteristics caused by PAX5 haploid deletion were different from that caused by PAX5 diploid deletion. PAX5 haploinsufficiency has been reported as being frequently altered in adult B-ALL [7]. Analysis of genetic variation characteristics caused by
PAX5 haploid deletion was very important for clarifying the role of PAX5 haploid deletion in tumorigenesis. We analyzed mRNA expression profile from two PAX5+/- Raji cell lines; only 135 common target genes were selected. The analysis of PPI Networks found that there were only 49 genes which were related to each other. These genes were divided into two networks: one network was interlocking histone genes clusters and another network consisted of 39 genes, which included several important node genes such as EGFR, FOS, HSPA5, TLR4, and MMP9 (Figure 3).

DAVID functional clustering of 49 genes returned 18 clusters, and there were 5 clusters with enrichment scores 1 . These significant clusters reflected that the dataset was representative of basic biological processes. These processes included gene transcription, inflammatory and immune response, tumor pathway, cell adhesion, and tight junction.

The processes where transcription factors regulate target genes are complicated, and histones play important roles during these processes. Histone gene clusters are heterogeneously organized and contain 1 or more copies of the 5 histone subtypes, that is, core $(\mathrm{H} 2 \mathrm{~A}, \mathrm{H} 2 \mathrm{~B}, \mathrm{H} 3, \mathrm{H} 4$, and variants thereof) and linker (H1) histone genes [24]. Histones determine target genes expression, and histone mRNAs are closely regulated during the cell cycle, permitting the synthesis of histone proteins to occur coordinately with the replication of DNA. Our results showed that 12 histone genes were all upregulated; these genes were all related to gene transcription. In tumors, upregulation of histone mRNA indicates proliferative activity of tumor cells. Many studies have demonstrated that histone mRNA accumulates in tumors [25-28] and histones control target genes expression. These histones may take part in the process where PAX5 haploinsufficiency causes gene expression changes. In our identified histones, previous literatures showed that HIST1H2BF, HIST1H3B, HIST1H4C, and HIST1H3D took part in tumorigenesis [28-31]. Many previous researches showed that other genes related to gene transcription were involved in tumorigenesis and prognosis [32-34]. Chromatin modifications implicated in transcriptional regulation are 
thought to be the result of a code on the histone proteins [35]. Abnormal expression of histone genes caused genomic instability, which enabled cells to acquire genetic alterations that promoted oncogenesis [36]. We guessed that the role of PAX5 haploinsufficiency in tumorigenesis might be partially achieved by PAX5 haploinsufficiency inducing genomic instability, although there was no report about PAX5 haploinsufficiency inducing genomic instability.

PAX5 is a member of the PAX family of developmental transcription factors with an important role in B-cell development. PAX5 activates the chromatin of key genes involved in B-cell signaling, adhesion, migration, and immune function [37]. The genes involved in inflammatory and immune response included TICAM2, CCL4L2, TLR4, FOS, AKT3, and PTPN6. These genes took part in tumorigenesis [3842]. TLR4, Fos, and AKT3 were primary node genes. PAX5 haploinsufficiency induced decreased expression of TLR4 and Fos and induced increased expression of AKT3. TLRs play important roles in regulating innate immune responses. TLR4 controls the host defense by sensing an exotic pathogen. TLR4 is often overexpressed in malignant and tumor-infiltrating immune cells, and the application of TLR4 ligands in cancer therapies is desirable for enhancement of antitumor immunity [38, 39]. PAX5 haploinsufficiency induced decreased expression of TLR4, and the downregulated TLR4 participated in immune tolerance [43, 44]. Fos was involved in inducing a large number of cytokine genes and other genes that were central to the productive immune response. The absence of Fos induced immune tolerance $[45,46]$. AKT3 is involved in a variety of biological processes. Highly expressed AKT3 participated not only in tumorigenesis [47] but also in autoimmune encephalomyelitis and graftversus-host response $[40,48]$. No study was reported about PAX5 haploinsufficiency involved in immune tolerance, but we had evidence to assume that PAX5 haploinsufficiency may result in tumor immune tolerance.

Cancer pathways participated in cancer pathophysiological processes. PAX5 haploinsufficiency caused the abnormal expression of genes involved in cancer pathways. Increased genes included EGFR, FGF12, AKT3, and CTNNA3 (Figure 3(c), Table 1). EGFR is a transmembrane tyrosine kinase receptor involved in the regulation of cellular multiplication, survival, and differentiation. EGFR is identified as cellular protooncogene and is overexpressed in a variety of human cancers [49]. EGFR inhibitors were used to treat not only non-small-cell lung cancer with EGFR mutation, but also cancer with overexpression of EGFR [50]. FGF12, as one proapoptotic gene, suppresses radiation-induced apoptosis through p38 $\alpha[51,52]$. FGF12 was identified as a new potential marker for prostate tumors [53, 54]. AKT, a major downstream mediator of $\mathrm{PI} 3 \mathrm{~K}$ pathway, was shown to regulate cancer progression. Three highly homologous AKT isoforms (i.e., AKT1, AKT2, and AKT3) may play different roles. Increased AKT3 expression not only promoted prostate cancer proliferation [55], but also conferred resistance to AKT inhibitor in breast cancer [56] and PLK inhibitors in human colorectal cancer [57]. Inhibiting AKT3 and PI3KCA enhanced chemotherapy sensitivity in glioblastoma multiforme cells [47].

\section{Conclusion}

In conclusion, our study here shows that changes in the gene transcription, inflammatory and immune response, and cancer pathways were identified as three important pathways associated with PAX5 haploinsufficiency. These changes hinted that the mechanism of PAX5 haploinsufficiency promoting tumorigenesis may be related to genomic instability, immune tolerance, and tumor pathways.

\section{Competing Interests}

The authors declare that there are no competing interests regarding the publication of this paper.

\section{Acknowledgments}

This work was supported by a grant from the National Natural Science Foundation of China (no. 81370658).

\section{References}

[1] B. M. Christopher, C. G. Mullighan, X. Su et al., "Pax5 haploinsufficiency cooperates with BCR-ABL1 to induce acute lymphoblastic leukemia," Blood, vol. 112, article 293, 2008.

[2] C. G. Mullighan, S. Goorha, I. Radtke et al., "Genomewide analysis of genetic alterations in acute lymphoblastic leukaemia," Nature, vol. 446, no. 7137, pp. 758-764, 2007.

[3] J. Medvedovic, A. Ebert, H. Tagoh, and M. Busslinger, "Pax5: a master regulator of B cell development and leukemogenesis," Advances in Immunology, vol. 111, pp. 179-206, 2011.

[4] P. O'Brien, P. Morin Jr., R. J. Ouellette, and G. A. Robichaud, "The Pax-5 gene: a pluripotent regulator of B-cell differentiation and cancer disease," Cancer Research, vol. 71, no. 24, pp. 73457350, 2011.

[5] C. Cobaleda, W. Jochum, and M. Busslinger, "Conversion of mature B cells into T cells by dedifferentiation to uncommitted progenitors," Nature, vol. 449, no. 7161, pp. 473-477, 2007.

[6] L. M. Heltemes-Harris, M. J. L. Willette, L. B. Ramsey et al., "Ebf1 or Pax5 haploinsufficiency synergizes with STAT5 activation to initiate acutelymphoblastic leukemia," Journal of Experimental Medicine, vol. 208, no. 6, pp. 1135-1149, 2011.

[7] I. Iacobucci, A. Lonetti, F. Paoloni et al., "The PAX5 gene is frequently rearranged in BCR-ABL1-positive acute lymphoblastic leukemia but is not associated with outcome. a report on behalf of the GIMEMA acute leukemia working party," Haematologica, vol. 95, no. 10, pp. 1683-1690, 2010.

[8] M. Kim, J. E. Choi, C. J. She et al., "PAX5 deletion is common and concurrently occurs with CDKN2A deletion in B-lineage acute lymphoblastic leukemia," Blood Cells, Molecules, and Diseases, vol. 47, no. 1, pp. 62-66, 2011.

[9] A. Liso, D. Capello, T. Marafioti et al., "Aberrant somatic hypermutation in tumor cells of nodular-lymphocyte- predominant and classic Hodgkin lymphoma," Blood, vol. 108, no. 3, pp. 10131020, 2006.

[10] G. Gaidano, L. Pasqualucci, D. Capello et al., "Aberrant somatic hypermutation in multiple subtypes of AIDS-associated nonHodgkin lymphoma," Blood, vol. 102, no. 5, pp. 1833-1841, 2003.

[11] M. Montesinos-Rongen, D. Van Roost, C. Schaller, O. D. Wiestler, and M. Deckert, "Primary diffuse large B-cell lymphomas of the central nervous system are targeted by aberrant 
somatic hypermutation," Blood, vol. 103, no. 5, pp. 1869-1875, 2004.

[12] M. Bousquet, C. Broccardo, C. Quelen et al., "A novel PAX5ELN fusion protein identified in B-cell acute lymphoblastic leukemia acts as a dominant negative on wild-type PAX5," Blood, vol. 109, no. 8, pp. 3417-3423, 2007.

[13] J. Familiades, M. Bousquet, M. Lafage-Pochitaloff et al., "PAX5 mutations occur frequently in adult B-cell progenitor acute lymphoblastic leukemia and PAX5 haploinsufficiency is associated with BCR-ABL1 and TCF3-PBX1 fusion genes: a GRAALL study," Leukemia, vol. 23, no. 11, pp. 1989-1998, 2009.

[14] J. Dang, L. Wei, J. De Ridder et al., "PAX5 is a tumor suppressor in mouse mutagenesis models of acute lymphoblastic leukemia," Blood, vol. 125, no. 23, pp. 3609-3617, 2015.

[15] M. A. J. Prasad, J. Ungerbäck, J. Åhsberg et al., "Ebf1 heterozygosity results in increased DNA damage in pro-B cells and their synergistic transformation by Pax5 haploinsufficiency," Blood, vol. 125, no. 26, pp. 4052-4059, 2015.

[16] C. Pridans, M. L. Holmes, M. Polli et al., "Identification of Pax5 target genes in early B cell differentiation," Journal of Immunology, vol. 180, no. 3, pp. 1719-1728, 2008.

[17] S. L. Nutt, S. Vambrie, P. Steinlein et al., "Independent regulation of the two Pax5 alleles during B-cell development," Nature Genetics, vol. 21, no. 4, pp. 390-395, 1999.

[18] D. Szklarczyk, A. Franceschini, M. Kuhn et al., "The STRING database in 2011: functional interaction networks of proteins, globally integrated and scored," Nucleic Acids Research, vol. 39, no. 1, pp. D561-D568, 2011.

[19] P. Shannon, A. Markiel, O. Ozier et al., "Cytoscape: a software environment for integrated models of biomolecular interaction networks," Genome Research, vol. 13, no. 11, pp. 2498-2504, 2003.

[20] D. W. Huang, B. T. Sherman, and R. A. Lempicki, "Systematic and integrative analysis of large gene lists using DAVID bioinformatics resources," Nature Protocols, vol. 4, no. 1, pp. 44-57, 2009.

[21] M. Kanehisa, S. Goto, M. Furumichi, M. Tanabe, and M. Hirakawa, "KEGG for representation and analysis of molecular networks involving diseases and drugs," Nucleic Acids Research, vol. 38, no. 1, pp. D355-D360, 2010.

[22] J. D. Sander and J. K. Joung, "CRISPR-Cas systems for editing, regulating and targeting genomes," Nature Biotechnology, vol. 32, no. 4, pp. 347-350, 2014.

[23] S. L. Nutt, B. Heavey, A. G. Rolink, and M. Busslinger, "Commitment to the B-lymphoid lineage depends on the transcription factor Pax5," Nature, vol. 401, no. 6753, pp. 556-562, 1999.

[24] W. F. Marzluff, P. Gongidi, K. R. Woods, J. Jin, and L. J. Maltais, "The human and mouse replication-dependent histone genes," Genomics, vol. 80, no. 5, pp. 487-498, 2002.

[25] M. Piscopo, G. Campisi, G. Colella et al., "H3 and H3.3 histone mRNA amounts and ratio in oral squamous cell carcinoma and leukoplakia," Oral Diseases, vol. 12, no. 2, pp. 130-136, 2006.

[26] R. Sakamoto, T. Nieta, Y. Kamikawa et al., "The assessment of cell proliferation during 9,10-dimethyl-1,2-benzanthraceneinduced hamster tongue carcinogenesis by means of histone $\mathrm{H} 3$ mRNA in situ hybridization," Medical Electron Microscopy, vol. 37, no. 1, pp. 52-61, 2004.

[27] M. D. Smith, E. Healy, V. Thompson, A. Morley, and J. L. Rees, "Use of in situ detection of histone mRNA in the assessment of epidermal proliferation: comparison with the Ki67 antigen and BrdU incorporation," British Journal of Dermatology, vol. 132, no. 3, pp. 359-366, 1995.
[28] T. Iwaya, T. Fukagawa, Y. Suzuki et al., "Contrasting expression patterns of histone mRNA and microRNA 760 in patients with gastric cancer," Clinical Cancer Research, vol. 19, no. 23, pp. 6438-6449, 2013.

[29] L. Rieswijk, S. M. H. Claessen, O. Bekers et al., "Aflatoxin B1 induces persistent epigenomic effects in primary human hepatocytes associated with hepatocellular carcinoma," Toxicology, vol. 350-352, pp. 31-39, 2016.

[30] S. Ryall, R. Krishnatry, A. Arnoldo et al., “Targeted detection of genetic alterations reveal the prognostic impact of H3K27M and MAPK pathway aberrations in paediatric thalamic glioma," Acta Neuropathologica Communications, vol. 4, no. 1, 2016.

[31] J. A. Han, J. Kim, and J. Kim, "Analysis of gene expression in cyclooxygenase-2-overexpressed human osteosarcoma cell lines," Genomics \& Informatics, vol. 12, no. 4, pp. 247-253, 2014.

[32] H. Guan, Z. Dai, Y. Ma, Z. Wang, X. Liu, and X. Wang, "MicroRNA-101 inhibits cell proliferation and induces apoptosis by targeting EYA1 in breast cancer," International Journal of Molecular Medicine, vol. 37, no. 6, pp. 1643-1651, 2016.

[33] X. Feng, S. Jia, T. A. Martin, and W. G. Jiang, "Regulation and involvement in cancer and pathological conditions of MAGI1, a tight junction protein," Anticancer Research, vol. 34, no. 7, pp. 3251-3256, 2014.

[34] S. H. Kim, J.-N. Ho, H. Jin et al., "Upregulated expression of BCL2, MCM7, and CCNE1 indicate cisplatin-resistance in the set of two human bladder cancer cell lines: T24 cisplatin sensitive and T24R 2 cisplatin resistant bladder cancer cell lines," Investigative and Clinical Urology, vol. 57, no. 1, pp. 63-72, 2016.

[35] S. Misri, S. Pandita, R. Kumar, and T. K. Pandita, “Telomeres, histone code, and DNA damage response," Cytogenetic and Genome Research, vol. 122, no. 3-4, pp. 297-307, 2008.

[36] K. Yoshioka, Y. Atsumi, H. Nakagama, and H. Teraoka, "Development of cancer-initiating cells and immortalized cells with genomic instability," World Journal of Stem Cells, vol. 7, no. 2, pp. 483-489, 2015.

[37] A. Schebesta, S. McManus, G. Salvagiotto, A. Delogu, G. A. Busslinger, and M. Busslinger, "Transcription factor Pax5 activates the chromatin of key genes involved in B cell signaling, adhesion, migration, and immune function," Immunity, vol. 27, no. 1, pp. 49-63, 2007.

[38] S. Ran, "The role of TLR4 in chemotherapy-driven metastasis," Cancer Research, vol. 75, no. 12, pp. 2405-2410, 2015.

[39] Y. Maru, T. Tomita, A. Deguchi et al., "Drug targeting based on a new concept-targeting against TLR4 as an example," Endocrine, Metabolic and Immune Disorders-Drug Targets, vol. 15, no. 2, pp. 83-87, 2015.

[40] W. Sang, C. Sun, C. Zhang et al., "MicroRNA-150 negatively regulates the function of $\mathrm{CD} 4+\mathrm{T}$ cells through AKT3/Bim signaling pathway," Cellular Immunology, vol. 306-307, pp. 3540, 2016.

[41] G. Youssef, C. Gillett, O. Agbaje, T. Crompton, and X. Montano, "Phosphorylation of NTRK1 at Y674/Y675 induced by TP53dependent repression of PTPN6 expression: a potential novel prognostic marker for breast cancer," Modern Pathology, vol. 27, no. 3, pp. 361-374, 2014.

[42] S. Wang, X. Xu, F. Xu et al., "Combined expression of c-jun, cfos, and p53 improves estimation of prognosis in oral squamous cell carcinoma," Cancer Investigation, vol. 34, no. 8, pp. 393-400, 2016.

[43] M. Xiang, J. Lu, C. Zhang et al., "Identification and quantification of total coumarins from Urtica dentata hand and its roles 
in promoting immune tolerance via TLR4-mediated dendritic cell immaturation," Bioscience, Biotechnology and Biochemistry, vol. 77, no. 6, pp. 1200-1206, 2013.

[44] M. Eguchi, Y. Sekiya, M. Suzuki, T. Yamamoto, and H. Matsui, "An oral Salmonella vaccine promotes the down-regulation of cell surface Toll-like receptor 4 (TLR4) and TLR2 expression in mice," FEMS Immunology and Medical Microbiology, vol. 50, no. 3, pp. 300-308, 2007.

[45] H. Bendfeldt, M. Benary, T. Scheel et al., "Stable IL-2 decision making by endogenous c-Fos amounts in peripheral memory T-helper cells," Journal of Biological Chemistry, vol. 287, no. 22, pp. 18386-18397, 2012.

[46] F. Macián, F. García-Cózar, S.-H. Im, H. F. Horton, M. C. Byrne, and A. Rao, "Transcriptional mechanisms underlying lymphocyte tolerance," Cell, vol. 109, no. 6, pp. 719-731, 2002.

[47] M. Paul-Samojedny, A. Pudełko, M. Kowalczyk et al., "Combination therapy with AKT3 and PI3KCA siRNA enhances the antitumor effect of temozolomide and carmustine in T98G glioblastoma multiforme cells," BioDrugs, vol. 30, no. 2, pp. 129144, 2016.

[48] V. Tsiperson, R. C. Gruber, M. F. Goldberg et al., "Suppression of inflammatory responses during myelin oligodendrocyte glycoprotein-induced experimental autoimmune encephalomyelitis is regulated by AKT3 signaling," Journal of Immunology, vol. 190, no. 4, pp. 1528-1539, 2013.

[49] J. Yang, S. Li, B. Wang et al., "Potential biomarkers for antiEGFR therapy in metastatic colorectal cancer," Tumor Biology, vol. 37, no. 9, pp. 11645-11655, 2016.

[50] K. Nakai, M. C. Hung, and H. Yamaguchi, "A perspective on anti-EGFR therapies targeting triple-negative breast cancer," American Journal of Cancer Research, vol. 6, no. 8, pp. 16091623, 2016.

[51] F. Nakayama, K. Müller, A. Hagiwara, R. Ridi, M. Akashi, and V. Meineke, "Involvement of intracellular expression of FGF12 in radiation-induced apoptosis in mast cells," Journal of Radiation Research, vol. 49, no. 5, pp. 491-501, 2008.

[52] F. Nakayama, S. Umeda, T. Yasuda et al., "Cellular internalization of fibroblast growth factor-12 exerts radioprotective effects on intestinal radiation damage independently of FGFR signaling," International Journal of Radiation Oncology Biology Physics, vol. 88, no. 2, pp. 377-384, 2014.

[53] S. O. Vozianov, V. I. Kashuba, V. M. Grygorenko et al., "Identification of a new diagnostic markers of prostatic cancer, using noti-microchips," Klinichna Khirurhiia, no. 4, pp. 54-57, 2016.

[54] A. A. Dmitriev, E. E. Rosenberg, G. S. Krasnov et al., "Identification of novel epigenetic markers of prostate cancer by NotI-microarray analysis," Disease Markers, vol. 2015, Article ID 241301, 13 pages, 2015.

[55] H.-P. Lin, C.-Y. Lin, C. Huo et al., "AKT3 promotes prostate cancer proliferation cells through regulation of Akt, B-Raf \& TSC1/TSC2," Oncotarget, vol. 6, no. 29, pp. 27097-27112, 2015.

[56] C. Stottrup, T. Tsang, and Y. R. Chin, "Upregulation of AKT3 confers resistance to the AKT inhibitor MK2206 in breast cancer," Molecular Cancer Therapeutics, vol. 15, no. 8, pp. 19641974, 2016.

[57] Y. Nonomiya, K. Noguchi, N. Tanaka, T. Kasagaki, K. Katayama, and Y. Sugimoto, "Effect of AKT3 expression on MYC- and caspase-8-dependent apoptosis caused by polo-like kinase inhibitors in HCT 116 cells," Cancer Science, vol. 107, no. 12, pp. 1877-1887, 2016. 

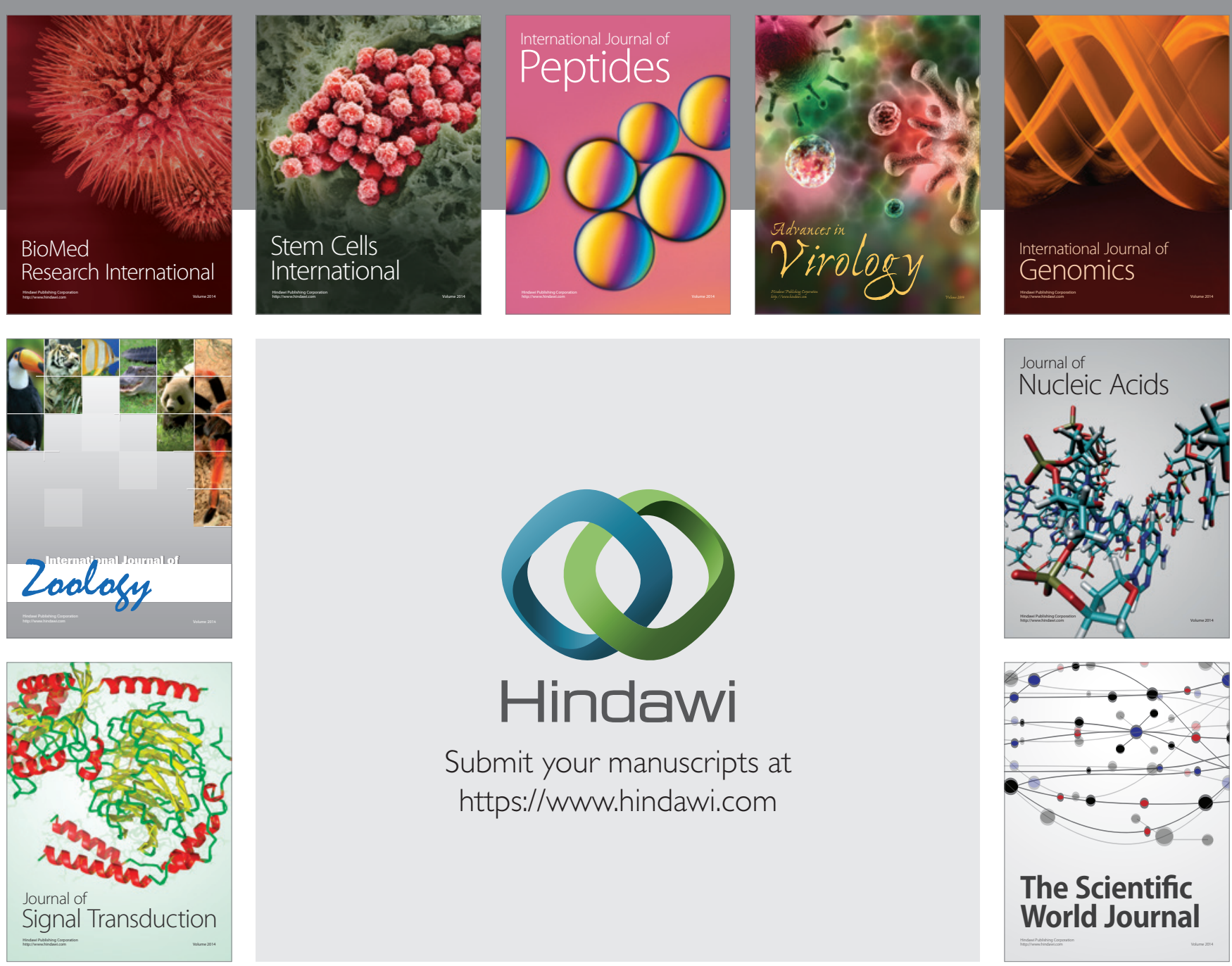

Submit your manuscripts at

https://www.hindawi.com
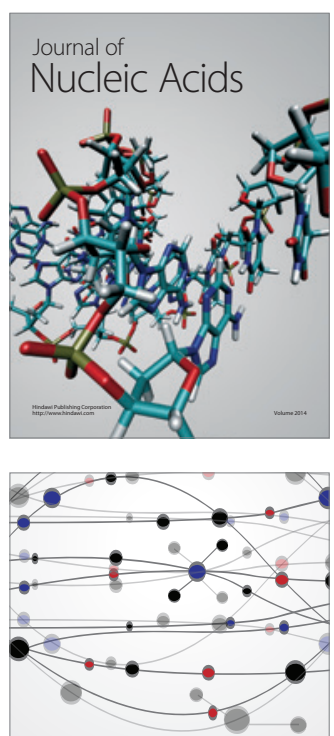

The Scientific World Journal
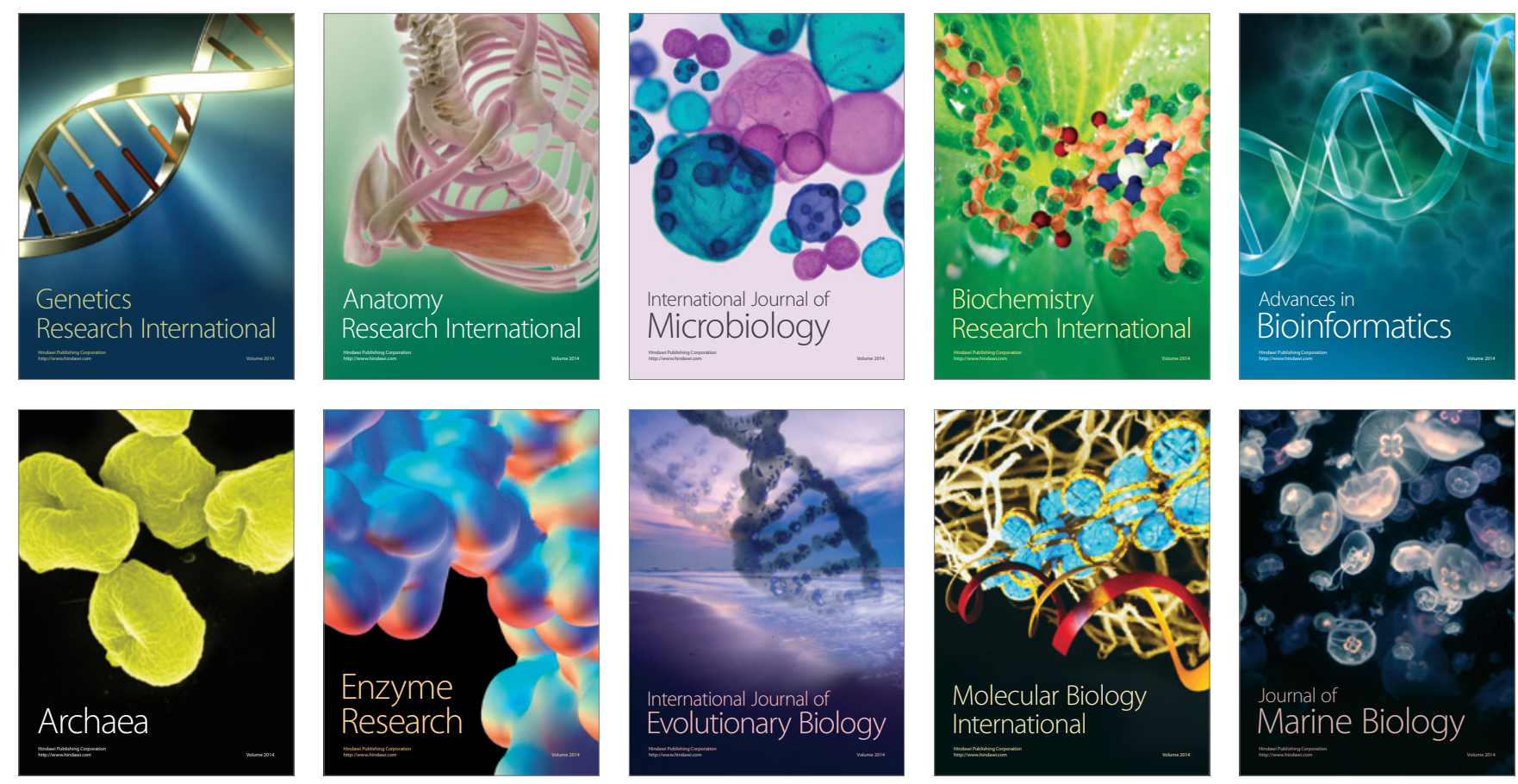